\title{
How Do Project-related Artefacts Qualify for Bridging Boundaries in IS Implementation Projects - An Activity Theoretical Perspective
}

\author{
Andy Weeger \\ Neu-Ulm University of Applied Sciences \\ andy.weeger@hs-neu-ulm.de
}

\begin{abstract}
Boundary objects were found to significantly impact the outcomes of IS implementation projects. Despite emphasizing their flexibility, however, prior research is no very precise on the attributes that qualifies project-related artefacts to become boundary objects. To identify the internal characteristics of artefacts that enable business and IT to synchronize IS and business needs, this research offers an activity theoretical view on boundary objects. The usefulness of the concept is demonstrated by means of an in-depth case study. The findings of this research emphasize that -in order to become boundary objects- projectrelated artefacts need to be part of the IS implementation and the business activity system. Moreover, they need to capture relevant knowledge of both activity systems and enable recognition of contradictions within and between. As to that, utilization of emerging project deliverables by means of internalization or externalization processes is found to facilitate the alignment between IS and business needs.
\end{abstract}

\section{Introduction}

Information system (IS) initiatives such as IS implementation projects usually intend to execute strategies by adapting systems and processes. Eventually, they aim to implement IS that is well aligned with strategic objectives and lead to positive business effects [1]. Social interactions between actors from different communities that enable the development of shared understanding are key for the success of these initiatives [2]. Such interactions usually take place between actors from the community that is concerned with making information technology work (i.e., IT) and from those that are utilizing IT within their activities to create value (i.e., the business).

Prior research indicates that the ability of IT and business to integrate specialized knowledge enables them to collaborate effectively and, ultimately, to align the requirements of the business and the abilities of the IS [see e.g. 3, 4]. In practice, however, there are various knowledge barriers that impede the communities to share and integrate their idiosyncratic knowledge and to develop shared understanding about the objectives of their initiatives $[5,6]$. To overcome these barriers, prior literature emphasizes the significance of project-related artefacts that bridge knowledge boundaries and thus enable knowledge sharing and cooperation across different communities of practice - so called boundary objects (BOs) [7, 8].

Hitherto, a large body of research has unveiled and established the importance of artefacts for crossboundary collaboration. In IS, much research aimed to better understand how project-related artefacts are used to bridge the business-IT boundary. In particular, prior research focused on how use-practices enable those artefacts to become BOs that help to convey and translate ideas between team members with diverse knowledge stocks [see e.g. 2, 5, 9, 10]. As to that, a huge variety of different objects in IS initiatives including enterprise systems [11], enterprise architectures [12], requirement specifications [5, 13], wireframes [14] and prototypes [2] were identified.

Despite the notion of flexibility and robustness, research was hitherto less concerned with the nature of artefacts utilized in IS initiatives and has thus not thoroughly captured the properties that enable them to bridge the business-IT boundary [9]. Rather, "the internal characteristics of BOs" have been "discussed on a general level” only [15, p. 8]. In practice, however, it is of particular importance to understand the aspects that enables artefacts to become BOs.

Aiming to increase our understanding on how learning between occupational communities involved in IS initiatives can be improved, this paper sets out to shed light on the inherent characteristics of BOs that facilitate knowledge integration between IT and business. Thus we put forth following research questions: How do project-related artefacts qualify as effective boundary-objects enabling business and IT to align IS and business needs?

To identify and to make sense of the internal characteristics of BOs employed in IS initiatives, the 
concept is complemented by cultural historical activity theory. The value of this complementary theoretical perspective is then illustrated by means of analysing data from an in-depth case study. To work out the characteristics that constitute the effectiveness of several project-related artefacts as BOs, the production and use of these artefacts during an IS implementation initiative are analysed. Ultimately, this paper outlines the essence of BOs that facilitate alignment between the IS and business needs and proposes several practical implications.

\section{Theoretical background}

\subsection{Boundary objects}

Communities of practice (CoP), at term coined by Wenger [8], share a joint area of concern, regularly interact within a set of community-specific norms and regulations, and draw on specialized pools of knowledge. These characteristics necessarily constitute boundaries between members of different CoPs [16], which can be distinguished between syntactic (e.g., syntax and langue differences), semantic (i.e., interpretive differences), and pragmatic (differences in goals in interests) knowledge boundaries [17]. BOs bridge these boundaries and enable interaction and coordination between members of different communities.

Wenger [8, p. 107] defines BOs as "artifacts, documents, terms, concepts, and other forms of reification around which communities of practice can organize their interconnections”. To act as common information spaces and to function as translation and transformation devices at the knowledge boundaries $[16,17,18]$, BOs need to be "plastic enough to adapt to local needs and constraints of the several parties employing them" and likewise "maintain a common identity across sites” [7, p. 393]. Thus, while members of different CoPs use and interpret BOs differently, these objects are useful for the work within the communities and the work that crosses the boundaries between them [7, 19]. Moreover, BOs are recognizable in different social worlds, enable individuals to re-contextualize local understandings in joint activities and provide common ground for communication and knowledge sharing [20].

In order to be effective, BOs must have several general representational characteristics [15]. First, BOs need to provide a shared language or syntax that allows idiosyncratic knowledge to be represented in a structure that is recognized on either side of the boundary [17]. Second, effective BOs provide "a concrete means for individuals to specify and learn about their differences and dependencies across a given boundary” and thus facilitate processes where actors can jointly transform their knowledge [17, p. 452]. Third, actors of multiple fields must be able to draw on and modify the content of a BO [17]. This also implies that, though providing a structure that is common to all involved groups, BOs are not static. Rather, BOs evolve as they are incorporated in local practices and modified to address internal or external contingencies [21].

IS research found a large array of artefacts that function as BOs, including business process diagrams [11], requirement specifications [5, 22], enterprise architecture designs and models [12], information systems [11, 13], and prototypes [2]. These artefacts can have different meanings within the work communities, while they are expected to provide a representational structure that is common to all these groups "so that they are recognizable to them and can serve as a means of translation" [23].

Analysing the role of these artefacts, prior IS research shows that artefacts, which function as BOs, are an important yet often overlooked condition for bridging syntactic, semantic and pragmatic knowledge boundaries between business and IT and within crossfunctional initiatives [13]. BOs were particularly found to facilitate knowledge integration and thus the development of shared understanding, required for aligning business needs, structures and processes and the abilities and limitation of IS [see e.g., 2, 5, 9, 24].

However, prior research is yet not very explicit about the constituents of the capability to maintain a common identity across sites in order to satisfy information and work requirements of business and IT, while being simultaneously employed in local practices. Particularly, little attention is given to the local and global context of BOs as well as the role of the multiple meanings the objects hold. Thus, there is potentially more learn about the nature of these objects, particularly the constituents of their ability to facilitate alignment between business and IT within cross-disciplinary initiatives such as IS projects.

Prior research reveals that using multiple theoretical perspectives enhances our understanding on the role of objects in cross-disciplinary collaboration [23]. Within this paper, the concept of BOs is complemented with the tenets of cultural historical activity theory (AT) to increase our understanding of the activities constituting the social worlds and how objects intersect these. Below AT and its primary concepts are briefly introduced.

\subsection{Activity theory}

Although AT is rather unfamiliar in IS research, it has emerged in related domains as an important theory 
for understanding change and development of ISmediated work activity [25, 26, 27, 28].

According to AT, a key characteristic of human activity is its object-oriented and mediated nature [29, 30]. Object-orientedness means that human activity is always directed towards a concrete or abstract entity that moves from potential 'raw material' or 'problem space' to a meaningful shape that forms the outcome of the activity [31, 32]. The object motivates the collective activity, determines individual goals and actions within, and only takes shape and acquires its value by means of the activity [32, 33].

To shape the object more efficiently, the subject of an activity -which may be an individual or a group of individuals- employs cognitive and materialized instruments [29, 34, 35]. These empower the subject with historically collected experience and skills [34]. Equally the instruments determine the possibilities and boundaries of their actions and interactions [36].

For instance, the common object of healthcare is the patient. Amongst others, the subject encompasses physicians and nurses. Their actions and interactions are directed towards the patients and mediated by tools such as a stethoscope or various ISs.

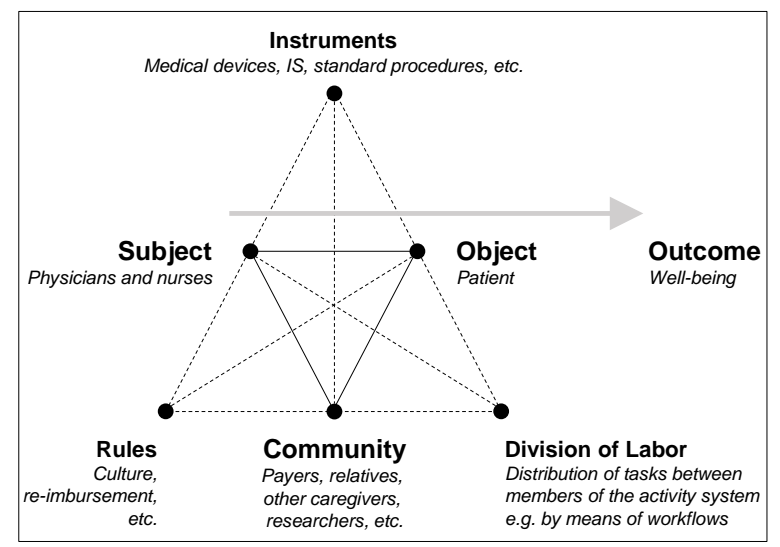

Figure 1. Activity system diagram

This instrument-mediated relationship between the subject and its object, however, only reflects 'the tip of an iceberg' [32]. The less visible mediators of the collective activity are the community, which encompasses all actors that revolve and evolve around the object and thus have a stake in the object, as well as rules and a division of labor, which inherently guide the actions and interactions of the subject and the community [23, 37]. The occurrence of rules and a division of labor indicates that the actions and interactions constituting collective activities are "always, explicitly or implicitly, characterized by ambiguity, surprise, interpretation, sense making, and potential for change” [31, p. 134]. Subsequently, AT emphasizes the complex and controversial nature of collective human activity, which is materialized in contested activity systems encompassing multiple mediating effects between subject, object, instruments, community, rules and the division of labor. These elements constitute the activity system. An example of such a system is depicted in Figure 1.

Considering healthcare, not only physicians and nurses are directed towards the patient, there is also a community encompassing, for instance, payers, relatives, other caregivers and researchers. The actions of actors are governed by regulations and implicit norms such as ethical principles as well as a complex division of labor, for instance, reflected in a stark hierarchy within hospitals. Although driving the activity, the patient as the object of healthcare activity may be considered as a person to be helped or as a source of revenue, for instance.

The contradictory, multi-voiced nature of human activity as assumed by AT explains why and how various tensions in groups or entire organizations emerge [38]. Referring to misfits "within elements, between them, between different activities, or between different developmental phases of a single activity”, contradictions are those forces that destabilize activities. They oppose "the overall motive of the system, the aim or purpose that subjects within the system are individually or collectively striving toward" [39, p. 840]. Although they "manifest themselves as problems, ruptures, breakdowns, and clashes” [28, p. 34], contradictions largely provide motives for subjecting the collective activity to change. Moreover, contradictions are seen as the driving force for the advancement of human activities [31]. Development can thus be seen as a process of resolving emergent and historically accumulated contradictions within and between these systems [40].

For instance, a contradiction between regulations that govern healthcare activities and the abilities of the tools employed may lead members of the activity systems to engage in a collective change effort. This change effort may aim at resolving the contradiction by enhancing the abilities of the instruments employed by virtue of a novel IS.

Moreover, activity systems must not be viewed in isolation, rather they can be understood as networks of interacting systems [35, 41]. Activities can adopt elements from the outside or may be dependent on the outcome of related systems. Although the modification of an element may aim at resolving emergent contradictions, introducing new or altering existing elements may also cause novel contradictions within or between the activity systems [40].

To increase understanding of organizational changes, AT and its concept of activity systems and contradictions as driver of change receives growing attention in IS research [39, 42, 43]. Inspired by these 
approaches, this study builds upon the above briefly outlined theoretical concepts and aims at enhancing our understanding on how project-related artefacts help to bridge knowledge boundaries between business and IT within IS implementation projects. Below, theoretical assumptions regarding the characteristics of BOs that facilitate knowledge integration between IT and business are derived.

\subsection{An activity theoretical view on BOs}

Building on the notion of interacting activity systems, the process of IS implementation can be understood as adapting elements of a specific activity (i.e., the business activity) by means of the outcomes of another activity (i.e., the IS implementation activity). Thus, IS implementation projects relate to two distinct, yet interrelated activity systems. Moreover, these systems constitute the boundaries of -at least- two distinct CoPs: the subject of the business activity (i.e., business) and the subject of the IS implementation activity (i.e., IT) [44].

The business activity aims to generate valuable outputs for the organization. For this purpose, the business utilizes instruments such as ISs. The ISs, in turn, are the output of IS implementation activities. During these activities, collaboration between the project team (i.e., subject) and business (i.e., parts of the community) is, for instance, governed by the project management and governance models (i.e., rules) and facilitated by project-related artefacts such as requirements specifications (i.e., instruments).

Implementing novel IS in business activities usually aims at aligning the toolset (i.e., instruments) and work practices (i.e., division of labor) with evolving strategies (i.e., rules). Building on AT's idea that development within activity systems is triggered by contradictions [40], IS implementation projects can be seen as a means to resolve contradictions within the business activity by adapting the instruments.

Although interrelated, the activities of business and IT are drawing on distinct, historically evolved knowledge that is mostly tacit, "socialized, embedded and invested in practice” [17, p. 442]. This knowledge is inscribed and materialized in rules, instruments, and the division of labor and therby helps the CoPs and their stakeholders to achieve their outcomes more efficiently. Moreover, the knowledge pools constitute the boundaries between the CoPs that need to be bridged in order to develop shared understanding about the contradictions and how the IS may resolve them (i.e., how to implement IS effectively) [9]. For this purpose, usually multiple instruments and projectrelated artefacts such as requirement elicitation methods, business process models and enterprise architecture designs are employed [5, 11, 12, 22]. From an AT perspective, particular candidates for BOs are those project-related artefacts and concepts that are utilized in the business and IS implementation activity and thus link both activity systems to a greater or lesser extent. Moreover, artefacts that link both activities are most-likely manipulated of the involved actors of both activities, for instance the object of IS implementation is transformed from a problem space (e.g., project goal) to a meaningful shape (e.g., prototypes). Implemented in both activities, such objects provide a structure that is recognized on either side of the boundary and that enables members of the distinct CoPs to learn about their activity systems.

Since implementing IS aims at resolving contradictions within the business activity, AT implies that BOs that enable business and IT to improve alignment between the IS and business needs must enable the translation of knowledge about key characteristics of the activity the IS is intended to be implemented within. As to that, BOs need to enable both CoPs to consistently interpret relevant parts of the business activity system (i.e., its subject, object, tool, community, rules and the division of labour) as well as the dependencies and dynamics within and between these elements. In particular, these objects need to allow the acting subjects of both activities to form explanations and expectations about how the IS will affect the activity system and how it need to be designed and implemented as to that it will resolve contradictions without creating novel ones.

In this regard, Engeström and Sannino [45, p. 371] state that "contradictions do not speak for themselves, they become recognized when practitioners articulate and construct them in words and actions". This implies that contradictions are to a significant extent manifested and constructed in discursive action. Thus, effective BOs must enable actors from involved CoPs to determine and discuss the multiple meanings of relevant parts of their activities. Ultimately, BOs must serve as magnets of the recognition of contradictions within the business activity system and enable involved CoPs to make sense of, deal with and resolve them by means of IS implementation.

However, BOs must also enable actors to bridge semantic and pragmatic boundaries regarding the limitations that the IS implementation activity imposes on the gestalt of its outcome. For instance, BOs must enable actors from IT and business to understand how the IS strategy governs the actions and interactions surrounding IS implementation.

Complementing the notion of BO with AT lead to following major assumptions regarding the attributes of BOs that enable business and IT to align the IS (abilities and characteristics) with business needs 
during IS implementation: (i) BOs need to be utilized in the activities of business and IT and thus bridge their activity systems; (ii) BOs need to facilitate translation of knowledge about key elements of the activity systems; (iii) BOs need to enable the distinct CoPs to recognize the contradictions aimed to resolve by means of IS implementation as well as contradictions that the novel IS may cause.

\section{Research design}

Recognizing a shortage of in-depth field studies considering BOs in IS projects [9] and the applicability of case study research for theory development and description, an in-depth analysis of a single case was selected $[46,47]$. As to that, a health IS implementation project is analyzed where knowledge boundaries between IT professionals and physicians were successfully bridged, while bridging boundaries between IT and healthcare was hardly successful. Both groups constitute the most relevant CoPs within hospitals. Examining this revelatory case is expected to facilitate the refinement of the initial theoretically grounded ideas and to illustrate the contributions of complementing the BO concept with activity theory [47]. Below a brief introduction to the case is given. Moreover, the methods employed to collect and analyze the data are presented.

\subsection{Case description}

The initiative analyzed in this paper aims to replace the patient data management system (PDMS), which is implemented in 15 intensive care and anesthesiology units (ICU) within a large German teaching hospital (hitherto O-PMDS). The approx. 20year-old systems ran out of life cycle and does not meet the obligations of the German medical product enactment. Moreover, physicians increasingly asked for advanced medical decision support functions that could not be implement using O-PDMS.

The management of the hospital thus decided to replace O-PDMS and initiated an interdisciplinary IS project with the primary objective to select and implement a successor PDMS that complies with the regulatory requirements and integrates well with the existing IT infrastructure. As to that, a project manager, four IT-consultants and representatives of physicians and nurses were assigned to the project. During all project phases, multiple artefacts have been employed in order to facilitate knowledge-integration between the project team, the vendor and representatives of key user at the wards (physicians and nurses).

\subsection{Data collection}

Data collection was approached by applying multiple methods such as semi-structured interviews, observations and conversations with stakeholders and users from the ICUs shortly before and after the rollout of the successor PDMS (November 2014 till February 2015).

Overall 16 formal interviews with 12 project stakeholders from IT (5) and business (7) across all hierarchical levels were conducted (e.g., project manager, project team members including IT consultants, nurses, nursing manager, ward physicians and assistant medical directors). These interviews were guided by an interview guideline that encompassed questions about each project phase. The interviews lasted 45 minutes at average (30 - 120 minutes). Almost all interviews could have been recorded (approx. $700 \mathrm{~min}$ audio recording) and transcribed (approx. 55.000 words).

Additional informal conversations were -like the interviews that could not be recorded- immediately written up in a research diary. Moreover, an array of project related-artefacts such as the high-level requirements document, requirements-worksheets and supplementary material, catalog of tickets/open issues as well as several minutes were collected and discussed with the informants.

\subsection{Data analysis}

Data analysis followed an iterative process of examining data, building and refining the theoretical assumptions. As to that, findings of earlier stages informed later stages and vice versa.

First, all data was carefully reviewed and projectrelated-artefacts identified. Then, all data that refer to characteristics of these artefacts and/or indicate how these artefacts were used to bridge knowledge boundaries between the project team (particularly IT staff), physicians and nurses at the wards as well as data that indicates consequences of these processes were coded (i.e., open coding) [46, 48]. This coding was particularly informed by activity theory (e.g. utilization within activity systems, reflection of activity systems, recognition of contradictions, etc.).

Second, a pattern coding approach was applied to analyze coded data and identify how characteristics of the BOs relate to the efficiency of knowledge integration and evolving project outcomes [48]. As to that, all data related to a distinct BO was analyzed and assessed whether and to what extent the BO helped to overcome knowledge boundaries. This also involved a careful analysis of how the object properties enabled stakeholders to identify emerging contradictions related to N-PDMS. 
Third, emergent themes were compared with the assumptions derived from prior literature. This was aimed to increase confidence in internal and external validity of the findings. Internal validity checks were concerned with reconsidering if the characteristics of BOs actually relate to the outcomes, while external validity checks verified if the findings of the in-depth case study can be generalized to the theoretical propositions [47].

\section{Findings}

Analyzing case data revealed three major projectrelated artefacts that prior research denoted as BOs: requirements specifications (excel-worksheet and related documents), prototypes and information systems (O-PDMS and N-PDMS). Below the characteristics of these artefacts and their impacts on knowledge integration processes between the CoPs as well as on the alignment between the PDMS and medical practices are discussed.

\subsection{O-PDMS}

According to our informants, the legacy system OPDMS was almost perfectly aligned with healthcare activities (i.e. few contradictions related to O-PDMS were recognizable). Thus, O-PDMS served as a template for requirements elicitation. During the preimplementation phase, the project team developed high-level requirements, particularly based on the functionalities of O-PDMS (instrument within the healthcare activity) and complemented by input of the user representatives (healthcare subjects) regarding novel functionalities. Based on these requirements and an ample and user-driven selection process, the $\mathrm{N}$ PDMS was chosen as successor of O-PDMS.

Before starting to implement the requirements and adapt N-PDMS to the hospital-specific needs, the vendor was asked to familiarize with O-PDMS and the way it is utilized by the nurses (key-users, healthcare subjects). However, since the vendor of N-PDMS also developed O-PDMS, he has only superficially considered the hospital-specific adjustments to OPDMS and the related usage-patterns in the ICUs. On the other hand, the project team did not invest considerable effort to get familiar with the basic configuration of N-PDMS, its limitations and possibilities. Rather, they assumed that, although the user interface changes and some additional functionalities are provided, N-PDMS would resemble the inherent logic of O-PDMS virtually one-to-one and thus, well integrate with the historically evolved rules and the division of labor at the wards.
Ultimately, this lead to significant issues in later stages of the project. Particularly, the project team, key-user representatives and vendors' staff (subject within the IS implementation activity) failed to overcome knowledge boundaries. As case study data indicates, actors were not able to identify interpretive differences regarding how O-PDMS is used within the hospital and N-PDMS' inherent logic (i.e., semantic and pragmatic boundaries). For instance, N-PDMS allows only for a standard start document for each patient, regardless of the ICU. However, at a later stage, this approach was found to "contradict the way tasks are distributed in the medical departments" (quote of a member of the project team), which could have surfaced if the vendor and the project team had used both systems as instruments during requirements elicitation and feasibility analysis.

Analyzing data from latter project stages reveals that O-PDMS was flexible enough to adapt to local needs of the activities of the vendor and the project team and maintained a common identity across sites. However, the project team and the vendor were not able to recognize contradictions within the object of the IS implementation activity before evolving project deliverables (N-PDMS) were found to contradict existing and historically evolved elements of the healthcare activity. Particularly, hardly recognized contradicting goals of the vendor (minimizing custom adaptations to N-PDMS) and the project team (conform N-PDMS to O-PDMS as closely as possible) obstructed the collective objective of the IS implementation activity: to align N-PDMS with the healthcare activity at the best.

Since actors did not use the systems as instruments enabling them to understand historically evolved characteristics of the activities at the ICUs as well as their relations to these aspects, relevant knowledge of the activity systems was not translated. Rather, semantic and pragmatic knowledge boundaries remained undiscovered and emerging contradictions between N-PDMS and, e.g., the existing division of labour within the medical activity did not surface.

\subsection{Requirements and prototypes}

At the beginning of the implementation phase, the project team arranged several workshops with representatives of all professional groups and hierarchical levels to elaborate the high-level requirements and the kind of adaptations to N-PDMS that are necessary to meet the requirements of the healthcare activities at the ICUs. The refined requirements were discussed and documented by means of multiple worksheets and supplementary PowerPoint slides. After the requirements have been 
specified and approved, vendor staff and the IT consultants (subjects of the implementation activity) begun to customize the N-PDMS. Progress and evolving issues were discussed during follow-up meetings. During the requirements-elicitation and customization process, all key-users were able to interact with and review prototypes of the IS.

The requirements-document was set up following a standard-template of the hospitals IT-department. Both, the requirements document and the prototypes can be viewed as early outcomes of the IS implementation activity -the object that gradually moves from a problem space to a more meaningful shape. Considering the healthcare activity, utilization of both project artefacts differs significantly between nurses and physicians. Physicians managed to imagine how the collective requirements will affect their actions within the healthcare activity, actively engaged in shaping the requirements as parts of the community within the IS implementation activity and integrated evolving prototypes for test purposes as an instrument in their healthcare activities. As a consequence, physicians and the IT professionals were able to translate knowledge about the key elements of the activity systems, could anticipate how N-PDMS will affect their actions within the healthcare activity and recognize how it will enable them to realize their goals. Particularly, engaging with the requirements document and the prototype helped the physicians to participate in conversations with the project team that brought out dilemmas stemming from differences in their understanding. Eventually, these interactions helped them to align the characteristics and abilities of the IS with their needs ( "That's just how it should look like”, a physician interacting with a prototype).

In stark contrast, the nurses were hardly able to imagine how the requirements will materialize and the novel IS will impact the actions and interactions that constitute the healthcare activity. For instance, nurses did not recognize that there is a lack of adequate rules that govern the use of the novel features (e.g., whether and when need which parameters to be documented) and that many of the requirements of the physicians impact the historically marked division of labour between them and the physicians (e.g., a shift of workload from the physicians to the nurses). On the one hand, this can be attributed to the fact that they had problems to conceive the differences between the legacy system and the novel IS in abstract discussions and representations, to realize emerging issues within the healthcare activity and to engage discursive actions to deal with and resolve the issues ( I I just don't see how this works", nurse in reaction to abstract discussions). On the other hand, nurses utilized the evolving prototypes to a significantly lesser extent for test purposes (requirements elicitation and prototyping was "physician-intensive", a nurse) and did hardly asses how the prototype and yet-to-be-implemented requirements affect the collective healthcare activity systems, particularly their actions and interactions. Although the prototypes were found to function as a magnet of the recognition of existing and potential contradictions on the part of the physicians, a lack of utilization on the nurses' side inhibited them to take full effect as BOs.

\subsection{N-PDMS}

Once all key requirements that were captured in the specification have finally been implemented, users were trained and N-PDMS was rolled out. During the roll out at the first ICU several contradictions between N-PDMS and other elements of the healthcare activity system surfaced as problems and breakdowns, particularly related to the actions conducted by nurses. For instance, using N-PDMS tremendously increased nurses' effort for documentation related actions. Though physicians were contented with N-PDMS, nurses tried to obstruct the roll-out. However, hospital management made clear that there is no way back and that the medical professionals need to implement $\mathrm{N}$ PDMS within their activities. Simultaneously, they extended the project by a refinement-phase, where the project team was asked to fix the most critical issues.

Within this phase, the project team discussed the most significant issues with the users, prioritized changes and implemented critical adaptations. Eventually, the healthcare activities, particularly nurses' actions and interactions stabilized and $\mathrm{N}$ PDMS could be transferred to standard operations. Case study data also indicates that the knowledge integration processes during the refinement-phase enabled medical stakeholders to solve some problems on their own by adjusting other elements of the healthcare activity (e.g., implementing novel rules regarding minimal documentation requirements).

During and after the role out, N-PDMS became ultimately an artefact that tightly couples the IS implementation activity (i.e., as object) and the healthcare activities (i.e., as instrument). On the one hand, medical professionals including nurses were forced to integrate N-PDMS as instrument within their activities. On the other hand, the directive of hospital management amplified the project-team's effort to adjust N-PDMS such that major contradictions within the medical activity could be resolved.

For instance, utilizing N-PDMS revealed an inherent contradiction within the object of healthcare activities that revolve around quality and efficiency of healthcare delivery. While collecting as much data as 
possible improves medical decision making and increases quality of care, collecting additional data also consumes scarce resources such as time of medical professionals.

Once implemented, N-PDMS served as a magnet of the recognition of these contradictions and thus drove discursive actions and knowledge integration between the IT professionals, the physicians, and the nurses. Ultimately, this enabled involved CoPs to agree on adjustments of N-PDMS and other elements of the medical activity system such as modes of the distribution of labor and novel rules. Although many breakdowns were not anticipated initially, the outcomes of the IS implementation activity eventually resolved emerging contradictions and tensions within the healthcare activity.

\section{Discussion}

Based on the reflections about BOs and activity theory and the empirical evidences of the single case study, following conclusions can be drawn regarding the attributes that qualify project-related artefacts to become effective BOs that help business and IT professionals to effectively align the IS with business needs, structures and work-processes:

First, artefacts need to link the activities of distinct CoPs. As the findings regarding O-PDMS, the requirements and N-PDMS reveal, the artefacts must be utilized in both activity systems, most likely as instrument in the business activity and as the object that is shaped during IS implementation. As to that, the utilization of evolving project deliverables particularly facilitates the alignment between the IS and business needs. These artefacts can be either implemented as a matter of fact (e.g., using a prototype within a realworld scenario) or by internalization mechanisms that enable people to try potential interactions with reality without performing actual manipulations with real objects (e.g., mental simulations). Second, utilized in both activity systems by means of externalization or internalization, these artefacts need to capture knowledge about key elements of the activity systems and their interrelations. Third, proving a common information space, these artefacts need to facilitate the recognition of the contradictions within and between the concerned activity systems. As to that, the artefact must enable the CoPs to identify and agree on the contradictions that are aimed to resolve by means of IS implementation and enable them to anticipate contradictions that might rise by means of IS implementation.

\subsection{Implications for theory and practice}

By using an activity theoretical lens on BOs, this paper offers, even though in the early stages, theoretically grounded explanations how and why artefacts become effective BOs in IS implementation projects. As to that, this study contributes to boundary object theory by providing an activity systems theory background for the characteristics of BOs. The findings go beyond the frequently cited need of interpretative flexibility and concretizes the structural properties that enable what Star [18] calls "the process of tacking back-and-forth between the ill-structured and well-structured aspects of the arrangements", which is necessary for interdisciplinary problemsolving. Moreover, applying AT to extend the notion of BOs confirms and extend prior literature stating that

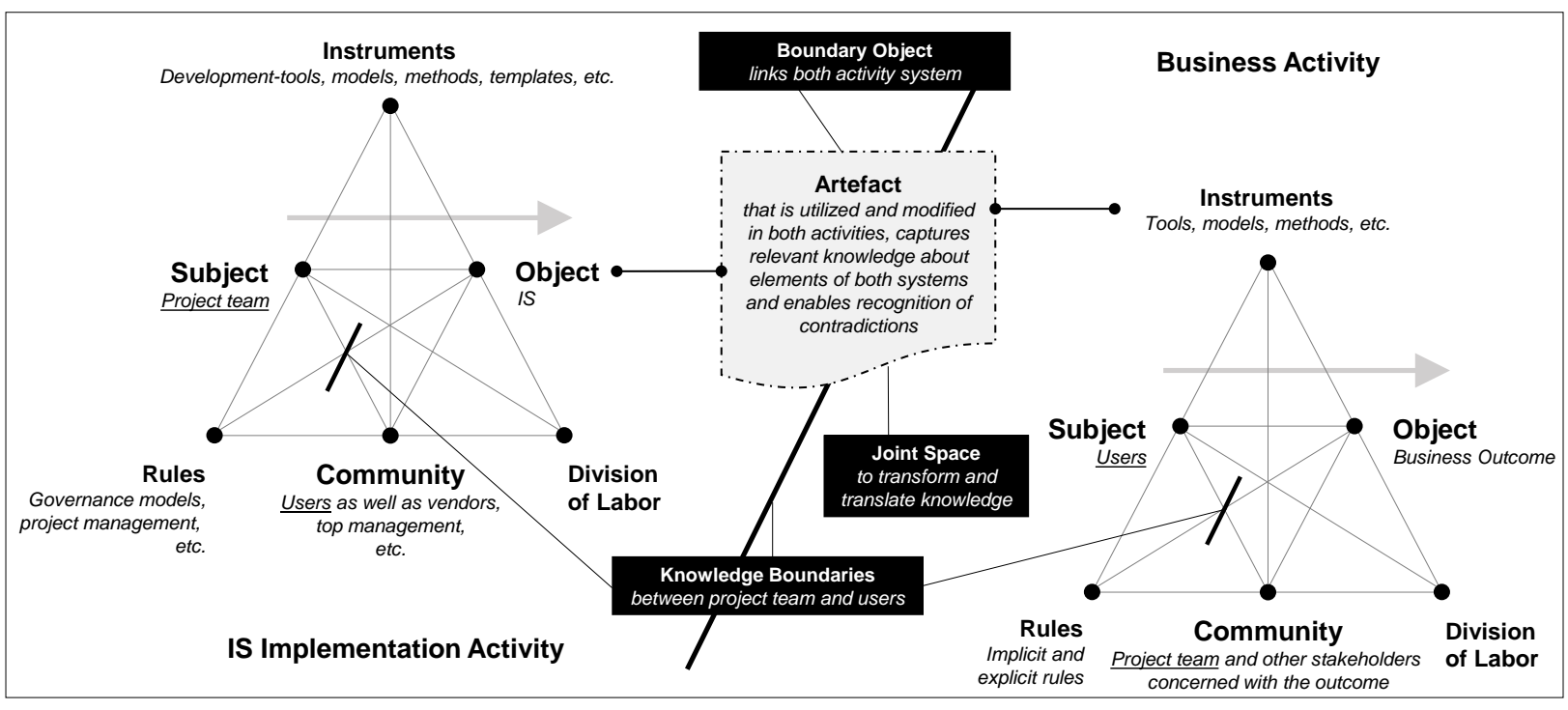

Figure 2. An activity theoretical view on boundary objects 
bridging knowledge boundaries requires not only persons but also objects to become legitimate participants in the activities of both CoPs [10]. Figure 2 summarizes the activity theoretical view on BOs in IS implementation as discussed in this paper.

From a practical stance, the findings of this study pave the way for the development of theoretically sound and actionable guidelines for shaping effective BOs that facilitate the alignment between the IS and business needs during such initiatives. For instance, practitioners may test to what extent rather abstract project artefacts such as requirements specifications enable people to try potential interactions with reality (i.e., internalization). As to that, these artefacts must enable CoPs to implement them in their activities -at least imaginary- and to anticipate how the novel IS will interact with the other elements of the activity, particularly how it will impact their actions and interactions with other members of the activity system.

Moreover, this research emphasizes the value of prototypes and practices like cooperative prototyping, in which IS are designed in part cooperatively by designers, prospective users and other stakeholders within IS implementation initiatives [49]. As a legitimate part of both activity systems, iteratively concretizing the prototype by means of agile methods, for instance, may facilitate the translation of knowledge of the key elements of the activity systems and enable IT and business to more efficiently align the IS that is implemented with business needs.

\subsection{Limitations}

As with all research, this study comes with limitations that need to be acknowledged. First, the data stem from a single case. Second, the case is embedded in a unique and rather complex domain. Third, case study data on early phases of the project could only be collected retrospectively. Further research employing multiple and longitudinal case studies may thus provide further evidence for the validity and usefulness of the activity theoretical perspective on BOs offered in this research. Moreover, such research may refine and expand these initial thoughts on objects that link activity systems and enable much needed knowledge translation and integration surrounding IS implementation initiatives. Moreover, the conclusions of this case study are only generalizable to the theoretical ideas derived from prior literature. Nonetheless, this study extends existing knowledge about BOs in IS implementation and provides valuable insights for contexts that extend this single case [46].

\section{References}

[1] Jenkin, T.A., and Chan, Y.E., "IS Project Alignment - a Process Perspective", Journal of Information Technology, 25(1), 2010, pp. 35-55.

[2] Doolin, B., and Mcleod, L., "Sociomateriality and Boundary Objects in Information Systems Development", European Journal of Information Systems, 21(5), 2012, pp. 570-586.

[3] Preston, D.S., and Karahanna, E., "Antecedents of IS Strategic Alignment: A Nomological Network", Information Systems Research, 20(2), 2009, pp. 159-179.

[4] Wagner, H.-T., Beimborn, D., and Weitzel, T., "How Social Capital among Information Technology and Business Units Drives Operational Alignment and IT Business Value", Journal of Management Information Systems, 31(1), 2014, pp. 241-272.

[5] Rosenkranz, C., Vranešić, H., and Holten, R., "Boundary Interactions and Motors of Change in Requirements Elicitation: A Dynamic Perspective on Knowledge Sharing", JAIS, 15(6), 2014, pp. 306-345.

[6] Tiwana, A., and Mclean, E.R., "Expertise Integration and Creativity in Information Systems Development", Journal of Management Information Systems, 22(1), 2005, pp. 13-43. [7] Star, S.L., and Griesemer, J.R., "Institutional Ecology, 'Translations' and Boundary Objects: Amateurs and Professionals in Berkeley's Museum of Vertebrate Zoology", Social Studies of Science, 19(3), 1989, pp. 387420.

[8] Wenger, E., Communities of Practice: Learning, Meaning, and Identity, Cambridge University Press, Cambridge, England, 1998.

[9] Winkler, M., Huber, T., and Dibbern, J., "The Software Prototype as Digital Boundary Object-a Revelatory Longitudinal Innovation Case", International Conference on Information Systems, 2014

[10] Levina, N., and Vaast, E., "The Emergence of Boundary Spanning Competence in Practice: Implications for Implementation and Use of Information Systems", MIS Quarterly, 29(2), 2005, pp. 335-363.

[11] Volkoff, O., Elmes, M.B., and Strong, D.M., "Enterprise Systems, Knowledge Transfer and Power Users", Journal of Strategic Information Systems, 13(4), 2004, pp. 279-304.

[12] Valorinta, M., "IT Alignment and the Boundaries of the IT Function", Journal of Information Technology, 26(1), 2010, pp. 46-59.

[13] Pawlowski, S.D., and Robey, D., "Bridging User Organizations: Knowledge Brokering and the Work of Information Technology Professionals", MIS Quarterly, 28(4), 2004, pp. 645-672.

[14] Levina, N., "Collaborating on Multiparty Information Systems Development Projects: A Collective Reflection-inAction View", Information Systems Research, 16(2), 2005, pp. 109-130.

[15] Ewenstein, B., and Whyte, J., "Knowledge Practices in Design: The Role of Visual Representations as `Epistemic Objects'", Organization Studies, 30(1), 2009, pp. 7-30.

[16] Carlile, P.R., "Transferring, Translating, and Transforming: An Integrative Framework for Managing Knowledge across Boundaries", Organization Science, 15(5), 2004, pp. 555-568. 
[17] Carlile, P.R., "A Pragmatic View of Knowledge and Boundaries: Boundary Objects in New Product Development", Organization Science, 13(4), 2002, pp. 442455.

[18] Star, S.L., "The Structure of Ill-Structured Solutions: Boundary Objects and Heterogeneous Distributed Problem Solving", in: Distributed Artificial Intelligence (Vol. 2)), Morgan Kaufmann Publishers Inc., 1989, pp. 37-54.

[19] Levina, N., and Vaast, E., "The Emergence of Boundary Spanning Competence in Practice: Implications for Implementation and Use of Information Systems", MIS Quarterly, 29(2), 2005, pp. 335-363.

[20] Bechky, B.A., "Sharing Meaning across Occupational Communities: The Transformation of Understanding on a Production Floor", Organization Science, 14(3), 2003, pp. 312-330.

[21] Subrahmanian, E., Monarch, I., Konda, S., Granger, H., Milliken, R., Westerberg, A., and Then-Dim, G., "Boundary Objects and Prototypes at the Interfaces of Engineering Design", Computer Supported Cooperative Work, 12(2), 2003, pp. 185-203.

[22] Levina, N., and Vaast, E., "Turning a Community into a Market: A Practice Perspective on Information Technology Use in Boundary Spanning", Journal of Management Information Systems, 22(4), 2006, pp. 13-37.

[23] Nicolini, D., Mengis, J., and Swan, J., "Understanding the Role of Objects in Cross-Disciplinary Collaboration", Organization Science, 23(3), 2012, pp. 612-629.

[24] Barrett, M., and Oborn, E., "Boundary Object Use in Cross-Cultural Software Development Teams", Human Relations, 63(8), 2010, pp. 1199-1221.

[25] Karanasios, S., Allen, D., and Finnegan, P., "Information Systems Journal Special Issue On: Activity Theory in Information Systems Research", Information Systems Journal, 25(3), 2015, pp. 309-313.

[26] Engeström, Y., "Activity Theory and the Social Construction of Knowledge: A Story of Four Umpires", Organization, 7(2), 2000, pp. 301-310.

[27] Jarzabkowski, P., "Strategic Practices: An Activity Theory Perspective on Continuity and Change", Journal of Management Studies, 40(1), 2003, pp. 23-55.

[28] Kuutti, K., "Activity Theory, Transformation of Work, and Information Systems Design", in: Perspectives on Activity Theory_Learning in Doing: Social, Cognitive and Computational Perspectives (Engeström, Y., Miettinen, R., and Punamäki-Gitai, R.-L., eds.), Cambridge University Press, Cambridge, UK, 1999, pp. 360-376.

[29] Leont'ev, A.N., Activity, Consciousness, and Personality, Prentice-Hall, Englewood Cliffs, New Jersey, 1978.

[30] Engeström, Y., "Objects, Contradictions and Collaboration in Medical Cognition: An ActivityTheoretical Perspective", Artificial Intelligence in Medicine, 7(5), 1995, pp. 395-412.

[31] Engeström, Y., "Expansive Learning at Work: Toward an Activity Theoretical Reconceptualization", Journal of Education and Work, 14(1), 2001, pp. 133-156.

[32] Engeström, Y., "Expansive Visibilization of Work: An Activity-Theoretical Perspective", Computer Supported Cooperative Work (CSCW), 8(1), 1999, pp. 63-93.
[33] Engestrom, Y., "On the Life of the Object", Organization, 12(3), 2005, pp. 307-330.

[34] Vygotsky, L.S.V., "Mind and Society: The Development of Higher Psychological Processes", Harvard University Press, Cambridge, MA, 1978

[35] Engeström, Y., Learning by Expanding. An ActivityTheoretical Approach to Developmental Research, OrientaKonsultit, Helsinki, 1987.

[36] Kuutti, K., "Activity Theory as a Potential Framework for Human-Computer Interaction Research", in: Context and Consciousness (Bonnie, A.N., ed.), Massachusetts Institute of Technology, 1995, pp. 17-44.

[37] Kaptelinin, V., "The Object of Activity: Making Sense of the Sense-Maker", Mind, Culture, and Activity, 12(1), 2005, pp. 4-18.

[38] Engeström, Y., "Communication, Discourse and Activity", The Communication Review, 3(1-2), 1999, pp. 165-185.

[39] Allen, D.K., Brown, A., Karanasios, S., and Norman, A., "How Should Technology-Mediated Organizational Change Be Explained? A Comparison of the Contributions of Critical Realism and Activity Theory", MIS Quarterly, 37(3), 2013, pp. 835-854.

[40] Cole, M., and Engeström, Y., "A Cultural Historic Approach to Distributed Cognition", in: Distributed Cognitions: Psychological and Educational Considerations (Salomon, G., ed.), Cambridge University Press, Cambridge 1993, pp. 1-46.

[41] Blackler, F., Crump, N., and Mcdonald, S., "Organizing Processes in Complex Activity Networks", Organization, 7(2), 2000, pp. 277-300.

[42] Karanasios, S., and Allen, D., "Mobile Technology in Mobile Work: Contradictions and Congruencies in Activity Systems", European Journal of Information Systems, 23(5), 2014, pp. 529-542.

[43] Karanasios, S., and Allen, D., "ICT for Development in the Context of the Closure of Chernobyl Nuclear Power Plant: An Activity Theory Perspective", Information Systems Journal, 23(4), 2013, pp. 287-306.

[44] Weeger, A., and Haase, U., "How Contradictions Facilitate Evolutionary Transformation: An Exploration into the Dynamics of Business-IT Alignment from the Perspective of Activi-Ty Theory", European Conference on Information Systems, 2016

[45] Engeström, Y., and Sannino, A., "Discursive Manifestations of Contradictions in Organizational Change Efforts", Journal of Organizational Change Management, 24(3), 2011, pp. 368-387.

[46] Yin, R.K., Case Study Research: Design and Methods, SAGE Publications, Thousand Oaks, CA, 2009.

[47] Eisenhardt, K.M., "Building Theories from Case Study Research", Academy of Management Review, 14(4), 1989, pp. 532-550.

[48] Miles, M.B., and Huberman, A.M., Qualitative Data Analysis: An Expanded Sourcebook, Sage Publications, Thousands Oaks, 1994.

[49] Mogensen, P., "Towards a Provotyping Approach in Systems Development", Scandinavian Journal of Information Systems, 4(1), 1992, pp. 31-53. 